\title{
Studying neurons and glia non-invasively via anomalous subdiffusion of intracellular metabolites
}

\author{
Carson Ingo ${ }^{1,2}$ (D) Wyger Brink ${ }^{3} \cdot$ Ece Ercan $^{4} \cdot$ Andrew G. Webb $^{3} \cdot$ Itamar Ronen $^{3}$
}

Received: 3 March 2018 / Accepted: 12 July 2018 / Published online: 7 August 2018

(c) The Author(s) 2018

\begin{abstract}
Cells in the central nervous system, neurons and glia, display a wide range of structural features. Molecular diffusion properties in the intracellular space of these cells reflect this structural diversity, deviating from standard Gaussian dynamics and resulting in anomalous subdiffusion. By tracking the displacement of intracellular metabolites, diffusion-weighted magnetic resonance spectroscopy allows for in vivo compartment-specific and cell-preferential morphological analysis of neurons and glia in the human brain. Suggestive of different intracellular environments between tissue type, the neuronal and glial intracellular space in gray matter is significantly more subdiffusive than in white matter. An important difference is found between the subdiffusion of choline, a predominantly glial metabolite, in gray and white matter, potentially reflecting differences in structural complexity between fibrous and protoplasmic astrocytes. The exclusively intracellular metabolite subdiffusive dynamics, taken together with water intra- and extracellular displacement, provide new insight of differing extracellular gray and white matter properties and exchange between tissue compartments.
\end{abstract}

Keywords Diffusion-weighted spectroscopy $\cdot$ Non-Gaussian diffusion · Cytomorphology $\cdot$ Neuron microstructure $\cdot$ Glia microstructure

Electronic supplementary material The online version of this article (https://doi.org/10.1007/s00429-018-1719-9) contains supplementary material, which is available to authorized users.

Carson Ingo

carson.ingo@northwestern.edu

Wyger Brink

w.m.brink@lumc.nl

Ece Ercan

ayse.ercan@utsouthwestern.edu

Andrew G. Webb

a.webb@lumc.nl

Itamar Ronen

i.ronen@lumc.nl

1 Department of Physical Therapy and Human Movement Sciences, Northwestern University, Chicago, IL, USA

2 Department of Neurology, Northwestern University, Chicago, IL, USA

3 Department of Radiology, C.J. Gorter Center for High Field MRI, Leiden University Medical Center, Leiden, The Netherlands

4 Department of Radiology, University of Texas Southwestern Medical Center, Dallas, TX, USA

\section{Introduction}

Cells such as neurons and glia in the mammalian central nervous system possess a wide range of structural and anatomical features which determine their individual as well as connected functions in the brain. This structural complexity can be visualized post-mortem using techniques such as high-resolution electron microscopy (Hayakawa et al. 2016; Matyash and Kettenmann 2010; Mobius et al. 2016; Oberheim et al. 2009; Pannese 2015). An in vivo method of analyzing this structural complexity and diversity is to use optical measurements of molecular diffusion properties in the intracellular space of these cells (Barkai et al. 2012). The heterogeneously crowded cytoplasm and intracellular complexity give rise to what is classified as anomalous subdiffusion, in which molecular displacement deviates from Gaussian dynamics and scales as a power law, reflecting a wide variation in diffusion rates (Banks and Fradin 2005; Ghosh et al. 2015; Weiss et al. 2004). This is true even in the extracellular space, for example, fluorescent-labeled molecules have been shown to exhibit subdiffusive dynamics in an excised rat cerebellum, supporting the notion that dead-space microdomains formed by astrocytic structures 
preferentially trap molecules which facilitates chemical exchange with the intracellular space (Xiao et al. 2015). To put it more simply, these observations of subdiffusion in cells describe molecular displacement as being slower than the simple Gaussian case of Brownian motion, being variable on the spatial or temporal scale, and being related to the complexity in the underlying biological microstructure.

Molecular diffusion can also be measured non-invasively and without the need for an exogenous tracer using magnetic resonance techniques, both imaging (MRI) and spectroscopy (MRS). The most common in vivo experiments involve diffusion-weighted imaging (DWI) of water in the human brain, where it has been well-established that there is a distribution of apparent diffusion coefficients, or diffusivities, within the neural tissue microstructure (Le Bihan et al. 1991). However, since water is non-specific to a particular tissue compartment or cell type, and exchanges between compartments, it is difficult to unequivocally determine how the differing cell types, and their cytoplasmic environments and widely different cellular morphologies, give rise to complex diffusion processes. In contrast to DWI, diffusion-weighted magnetic resonance spectroscopy (DWS) enables in vivo compartment-specific analysis of neurons and glia by measuring the bulk movement of intracellular metabolites such as total choline $(\mathrm{tCho}=$ choline + phosphocholine + glycerophosphocholine), total creatine ( $\mathrm{tCr}=$ creatine + phosphocreatine), and total N-acetyl-aspartate (tNAA $=\mathrm{NAA}+\mathrm{NAAgluta}-$ mate) specific to these cells (Cao and Wu 2016; Nicolay et al. 2001; Palombo et al. 2017; Ronen et al. 2014). Highresolution nuclear magnetic resonance (NMR) studies of brain extracts and cell cultures have shown cell-type specificity of the main metabolites which can be detected with ${ }^{1} \mathrm{H}-\mathrm{MRS}$ : choline compounds and myo-inositol are mainly found in astrocytes and oligodendrocytes, $\mathrm{N}$-acetyl-aspartate and glutamate are exclusively contained in neurons, while creatine and phosphocreatine are present in both glia and neurons (Choi et al. 2007; Le Belle et al. 2002; Urenjak et al. 1993). This feature of cell-specificity, in addition to the slow inter-compartmental exchange of these metabolites, makes their diffusion properties excellent probes of the structural and physiological properties of the intracellular space of neurons and glia. Over the last three decades, ${ }^{1} \mathrm{H}$ - and ${ }^{31} \mathrm{P}$ DWS studies have been carried out on excised neural tissue (Assaf and Cohen 1998a), as well as in vivo on muscle (Brandejsky et al. 2014; de Graaf et al. 2000; Moonen et al. 1990; van Doorn et al. 1996) and brain (Dreher et al. 2001; Marchadour et al. 2012; Merboldt et al. 1993; Pfeuffer et al. 2000; Valette et al. 2007; van der Toorn et al. 1996; Wick et al. 1995) in animal models. In recent years, ${ }^{1} \mathrm{H}$-DWS has been increasingly performed in humans, where the diffusion properties of the main brain metabolites $\mathrm{tCr}$, tCho and tNAA have been robustly and reproducibly measured in a variety of brain regions (Deelchand et al. 2017; Ellegood et al. 2005, 2006; Kroenke et al. 2004; Posse et al. 1993; Upadhyay et al. 2007, 2008). The ability of DWS to provide not only qualitative and descriptive information but also accurate quantitative in vivo microstructural information in the central nervous system has been shown in a number of publications (Kroenke et al. 2004; Marchadour et al. 2012; Najac et al. 2014; Palombo et al. 2016; Ronen et al. 2014; Shemesh et al. 2014).

In both DWI and DWS, diffusion sensitization is most commonly determined by the parameter $b$ which is a function of the strength of the applied diffusion gradients, the times they are applied for, and the time-separation between the applied gradients (Stejskal and Tanner 1965). At low $b$ values, typically $b<2000 \mathrm{~s} / \mathrm{mm}^{2}$ for DWI of water and $b<5000 \mathrm{~s} / \mathrm{mm}^{2}$ for DWS of intracellular metabolites, the decay of the diffusion-weighted signal in DWI and DWS in neural tissue is approximately monoexponential (Kan et al. 2012; Pierpaoli et al. 1996). For water, at higher $b$ values, the diffusion-weighted signal deviates from monoexponential decay and the diffusion process is then broadly described as non-Gaussian, with a number of interpretations and working models proposed for characterization (Assaf and Basser 2005; Hall and Barrick 2008; Jensen et al. 2005; Jones 2010; Le Bihan et al. 1991; Magin et al. 2008; Özarslan et al. 2012). Recently, this non-monoexponential behavior in water has been characterized as anomalous subdiffusion, a characterization that provides a theoretical link to optical tracing measurements in heterogeneously crowded cells (Ingo et al. 2014a, b, 2015; Metzler and Klafter 2000). Previous ex vivo DWS work has investigated the non-monoexponential diffusion-weighted signal for NAA in rat brain tissue (Assaf and Cohen 1998a) and bovine optic nerves (Assaf and Cohen 1998b). However, in vivo DWS studies have been confined to estimations of the apparent diffusion coefficient assuming a monoexponential shape in the diffusion signal at a single diffusion time (Ellegood et al. 2005; Kan et al. 2012) or at a multitude of diffusion times (Marchadour et al. 2012; Najac et al. 2014, 2016; Palombo et al. 2016) at relatively low $b$. A previous study reported on analyses of DWS data with a stretched exponential model (Branzoli et al. 2014) in which the range of $b$ sampled was relatively low $(<5000 \mathrm{~s} /$ $\mathrm{mm}^{2}$ ) and the results determined that the metabolite diffusion was close to monoexponential regardless of echo and mixing times $(\beta \sim 0.9)$.

This study presents results of DWS measurements performed on a 7 T MRI system over a wide range of $b$ up to about $17,000 \mathrm{~s} / \mathrm{mm}^{2}$ to characterize the non-monoexponential diffusion signals in white matter (WM) and gray matter (GM) of the healthy human brain in vivo. By performing experiments at a very high magnetic field we are able to acquire sufficient signal-to-noise to be able to use the very high $b$ values which are required to study the non-monoexponential behavior of metabolite diffusion in vivo, which could provide 
novel contrast between cell types independent of previous estimations of classical apparent diffusion coefficient. The first goal was to establish anomalous subdiffusive dynamics in the intracellular space of neurons and glia, and link them to the characterization of cell-type cytomorphological and cytoplasmic properties. The second goal was to use the information obtained on subdiffusive behavior of metabolites which reside exclusively in the intracellular space to shed light on the nature of water diffusion in both the intra- and extra-cellular compartments of brain tissue.

\section{Materials and methods}

\section{Human subjects}

A total of 22 volunteer subjects (age $24.7 \pm 3.9$ years) participated in this study. For the parietal WM volume of interest (VOI) acquisition, 12 volunteer subjects (age $25.4 \pm 2.8$ years, 6 female, 6 male) were scanned. For the occipital GM VOI acquisition, 10 volunteer subjects (age 23.9 \pm 4.9 years, 6 female, 4 male) were scanned. The study adhered to the Leiden University Medical Center Institutional Review Board guidelines and informed consent was obtained from all subjects prior to the study.

\section{MRI system, hardware, and maximization of $B_{1}$ transmission}

All experiments were performed on a $7 \mathrm{~T}$ Philips Achieva whole-body MRI scanner (Philips Healthcare, Best, Netherlands) equipped with gradient coils capable of a maximum gradient strength of $40 \mathrm{mT} / \mathrm{m}$ and a slew rate of $200 \mathrm{~T} / \mathrm{m} / \mathrm{s}$. A radiofrequency $(\mathrm{RF})$ head coil consisting of a quadrature birdcage transmit and 32-channel receive array was used for all measurements (Nova Medical Inc., Wilmington, MA, USA). A $15 \times 15 \times 1 \mathrm{~cm}^{3}$ high permittivity dielectric pad (suspension of barium titanate in deuterated water) was placed between the volunteer's head and the receive array for maximization of the transmit magnetic field sensitivity $\left(\mathrm{B}_{1}^{+}\right)$in the parietal and occipital VOIs (Brink et al. 2014; Teeuwisse et al. 2012). The RF transmit gain was calibrated to produce $90^{\circ}$ and $180^{\circ}$ flip angles within the spectroscopic VOI (Versluis et al. 2010; Webb and Collins 2010) by acquiring a wholehead $B_{1}$ map at a spatial resolution of $2.5 \times 2.5 \times 5 \mathrm{~mm}^{3}$ using the dual refocused acquisition mode (DREAM) sequence with TR/TE $=4.5 / 1.8 \mathrm{~ms}$ (Nehrke and Börnert 2012) (scan time $2 \mathrm{~min}$ ).

\section{MRI/MRS protocol}

\section{Anatomical images}

A short survey scan and a sensitivity encoding (SENSE) reference scan were followed by a $3 \mathrm{D} \mathrm{T}_{1}$-weighted gradient echo acquisition to allow for anatomical planning of subsequent scans, as well as tissue segmentation within the VOI. Imaging parameters were: field of view (anterior/posterior, foot/head, right/left): $246 \times 246 \times 174 \mathrm{~mm}^{3}$, resolution $1 \times 1 \times 1 \mathrm{~mm}^{3}, \mathrm{TR} / \mathrm{TE}=4.7 / 2.1 \mathrm{~ms}$ ( scan time $\left.\sim 7 \mathrm{~min}\right)$. The $\mathrm{T}_{1}$-weighted image was segmented into $\mathrm{WM}, \mathrm{GM}$, and CSF fractions using the FAST algorithm in the FMRIB Software Library (Jenkinson et al. 2012). The tissue probability maps from the FAST algorithm were used as an input to a custom written MATLAB routine to estimate the percentage of GM, WM and CSF within the two VOIs (Ercan et al. 2014).

\section{DWS}

Two VOIs were chosen: one in a subcortical parietal region containing mostly WM and one in a cortical region of the occipital lobe containing mostly GM. The parietal WM VOI was selected to minimize directional dependence of fiber orientation as has been shown for the diffusion properties in corpus callosum, for example (Ronen et al. 2013, 2014). Figure 1 shows the planned VOIs superimposed on $\mathrm{T}_{1}$-weighted images in three orthogonal views. VOI dimensions were $20 \mathrm{~mm}$ isotropic, giving a total volume of $8 \mathrm{~cm}^{3}$. Slight variations in positioning of the VOI across subjects were necessary to optimize local $\mathrm{B}_{1}$ sensitivity with respect to the placement of the dielectric pad relative to the head coil.

To allow for a sufficiently long diffusion time, a 13 interval stimulated echo-based pulsed gradient sequence ( $\mathrm{TE}=105 \mathrm{~ms}$ ) with bipolar diffusion gradients was used (Cotts et al. 1989). Cardiac synchronization on every 3rd cardiac cycle was implemented via a pulse-oximeter, resulting in a TR of about $3 \mathrm{~s}$, and was used to minimize strong fluctuations in signal intensity due to cardiac pulsation (Upadhyay et al. 2007). The number of time-domain points were 1024 and spectral width was $3000 \mathrm{~Hz}$. Static field shimming up to second order was performed using the vendor-supplied shimming routine, resulting in a typical water line width of about $12 \mathrm{~Hz}$. Three perpendicular directions were chosen to produce isotropic diffusion-weighting. To maximize the $b$ value for a given diffusion gradient strength, the directions were $[1,1,-0.5],[1,-0.5,1]$, and $[-0.5$, $1,1]$. Diffusion-weighting parameters were: gradient pulse duration $(\delta) 30 \mathrm{~ms}$, diffusion time $(\Delta) 100 \mathrm{~ms}$, bipolar delay ( $\tau) 13 \mathrm{~ms}, 11$ gradient amplitude values between 3 and 37 $\mathrm{mT} / \mathrm{m}$ resulting in a set of $b$ values of 402-17,204 s/mm at an effective diffusion time $(\Delta-\delta / 3-\tau / 2)$ of $83.5 \mathrm{~ms}$. Each 


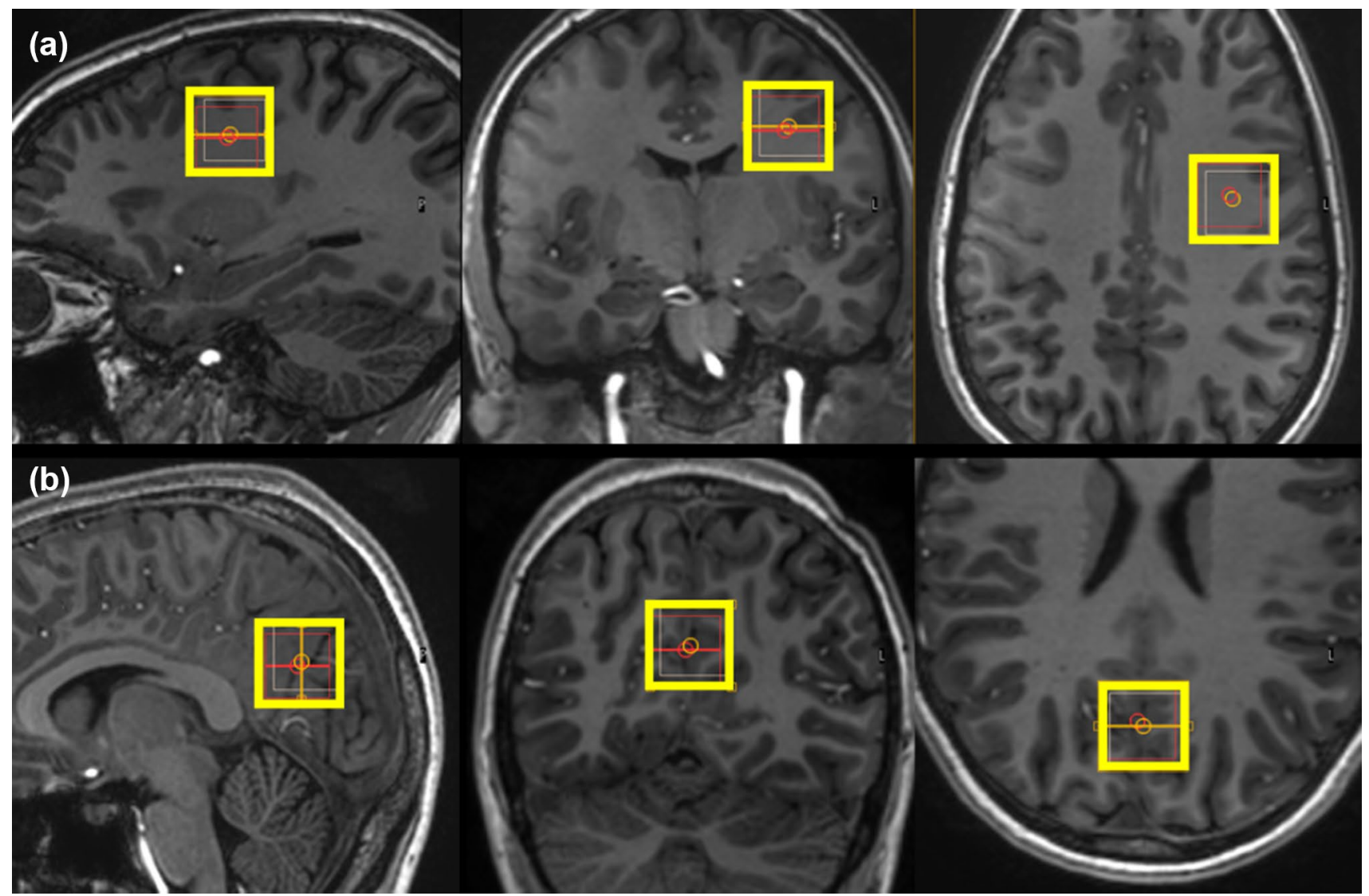

Fig. 1 Volumes of interest from which diffusion-weighted spectra are acquired, superimposed on sagittal, coronal, and transverse $\mathrm{T}_{1}$-weighted images for $\mathbf{a}$ the mostly white matter in a subcortical parietal region and $\mathbf{b}$ the mostly gray matter in a cortical region of the occipital lobe

of the 33 direction/gradient amplitude conditions (3 directions $\times 11$ gradient values) was acquired 16 times for a total set of 528 individual free induction decays (FIDs) (scan time $\sim 26 \mathrm{~min})$. The $b$ values were numerically calculated from the pulse sequence itself, which included the effective diffusion-weighting produced by the slab selection and crusher gradients.

"Weak" water suppression was performed by providing only a fraction of the radio frequency power needed for full water suppression to preserve enough of the water signal (about 5-10 times the magnitude of the NAA singlet for all conditions) for subsequent phasing and frequency drift correction of each individual acquisition. In the WM, between 85 and $91 \%$ (inter-subject variation) of the RF power for full water suppression was used for $b$ values less than $8000 \mathrm{~s} / \mathrm{mm}^{2}$ and $80-86 \%$ for higher $b$ values. For GM, between 82 and $90 \%$ of the RF power for full water suppression was used for $b$ values less than $5000 \mathrm{~s} / \mathrm{mm}^{2}$ and $70-74 \%$ for higher $b$ values. Additionally, a short acquisition with the same diffusionweighting conditions was performed without water suppression and subsequently used for water diffusion measurements and eddy current correction of the metabolite spectra. In this short water scan, each of the 33 direction/gradient amplitude conditions ( 3 directions $\times 11$ gradient values) was acquired 4 times for a total set of 132 individual free induction decays (scan time $\sim 6 \mathrm{~min}$ ).

\section{Preprocessing of diffusion weighted spectra}

DWS preprocessing was performed using a custom written program in Matlab (Mathworks Inc, Natick, MA, USA) as previously described (Kan et al. 2012). The main steps in the data processing included: weighted summation and phasing of the individual outputs of the 32 receive coils based on the reference water signal, eddy current correction, zero-order phase and frequency drift correction on each individual FID, followed by averaging of FIDs of each diffusion-weighted condition, and subsequent directional averaging to generate isotropic diffusion-weighted spectra, e.g. Fig. 2. The resulting spectra were analyzed using LCModel with an appropriate simulated set of basis spectra (Provencher 1993). 

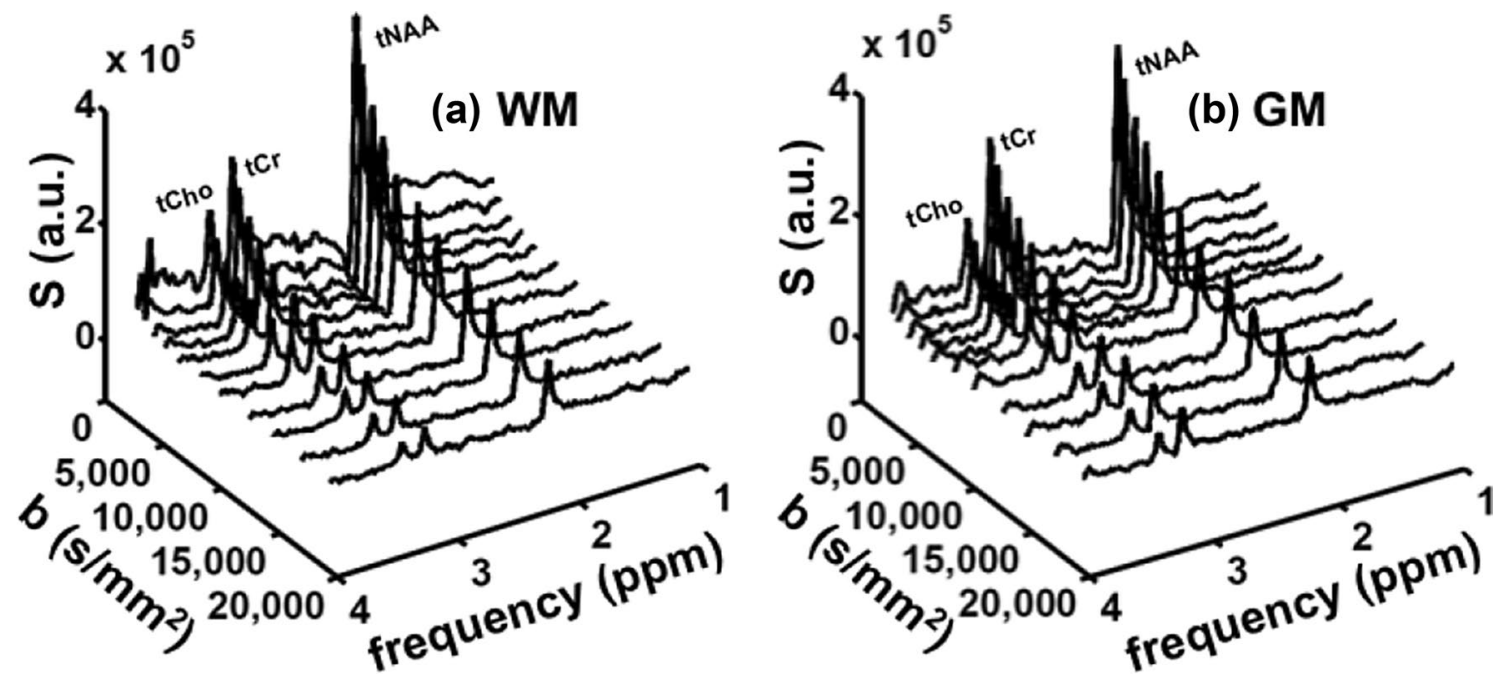

Fig. 2 Diffusion-weighted spectra showing the signal (S, arbitrary units) attenuation as a function of diffusion weighting ( $b$ value) in a the mostly white matter (WM) of the parietal lobe and $\mathbf{b}$ the mostly gray matter (GM) of the occipital lobe

\section{Diffusion-weighted signal modeling of metabolite and water data}

Following spectral quantification in LCModel, each metabolite and water diffusion-weighted signal was fitted to the Mittag-Leffler function (MLF) (Mittag-Leffler 1903, 1905) using previously published procedures (Ingo et al. 2015) and custom Matlab codes (Ingo et al. 2017), e.g. Fig. 3. Prior to minimum least-squared convergence to the MLF, a starting value for the classical diffusion coefficient, $D$, was estimated using a simple monoexponential function for metabolite data at $b<4000 \mathrm{~s} / \mathrm{mm}^{2}$ and water data at $b<1000 \mathrm{~s} / \mathrm{mm}^{2}$. Subsequently the starting value for $D$ and a starting value of $\alpha=1$ were used to estimate a final value of $D$ and the power law subdiffusion index, $0<\alpha \leq 1$ in Eq. 1 ,

$\frac{S(b)}{S(0)}=E_{\alpha}(-b D)$

where $E_{\alpha}$ is the single parameter MLF, which is the characteristic functional form derived from the fractional-order partial differential equation describing subdiffusion and power law dynamics (Ingo et al. 2015; Metzler and Klafter 2000). The MLF is a special function that corresponds to specific functions for particular values of $\alpha$ (Mittag-Leffler $1903,1905)$. For example, when $\alpha=1$, the MLF is the simple monoexponential function,

$E_{1}(-b D)=\exp (-b D)$.

Another case is when $\alpha=0.5$ and then MLF becomes the scaled complementary error function,

$E_{0.5}(-b D)=\exp \left(-(b D)^{2}\right) \operatorname{erfc}(b D)$.
Overall, $\alpha$ serves as a heterogeneity index to determine the deviation from homogeneous Gaussian diffusion $(\alpha \sim 1)$, and the smaller the value of $\alpha$, the more heterogeneous the diffusion, indicative of power law subdiffusive behavior.

\section{Monte Carlo simulations of DWS modeling results}

To determine the stability of the modeling results, $N=1000$ Monte Carlo simulations were performed on the diffusionweighted data utilizing the average values of the Cramér-Rao Lower bounds (CRLB) in the spectroscopic measurements for the value of the Gaussian noise variance at each condition for each metabolite.

\section{Results}

Table 1 shows the average WM, GM, and cerebrospinal fluid (CSF) percentages within the two VOIs, indicating an average WM fraction in the parietal VOI of about $72 \%$ and an average GM fraction of about $64 \%$ in the occipital VOI. While there was about $4-8 \%$ of average CSF in the VOIs, this percentage does not contribute to the diffusion results in this study as "free water" (Pasternak et al. 2009) only contributes to the diffusion-weighted signal at very low diffusion weightings (i.e., $b<500 \mathrm{~s} / \mathrm{mm}^{2}$ ) and does not contribute to the high $b$ water data or metabolite displacement estimates. Figure 2 shows pre-processed spectra (prior to LCModel quantification) as a function of the strength of the diffusion-weighting ( $b$ value), with the tCho, $\mathrm{tCr}$, and tNAA peaks identified according to their chemical shift in both the WM and GM VOIs. Figure 3 shows the measured signal intensities as a function of $b$ value, along with a line 
(a) WM b $\left.\quad \mathrm{s} / \mathrm{mm}^{2}\right)$
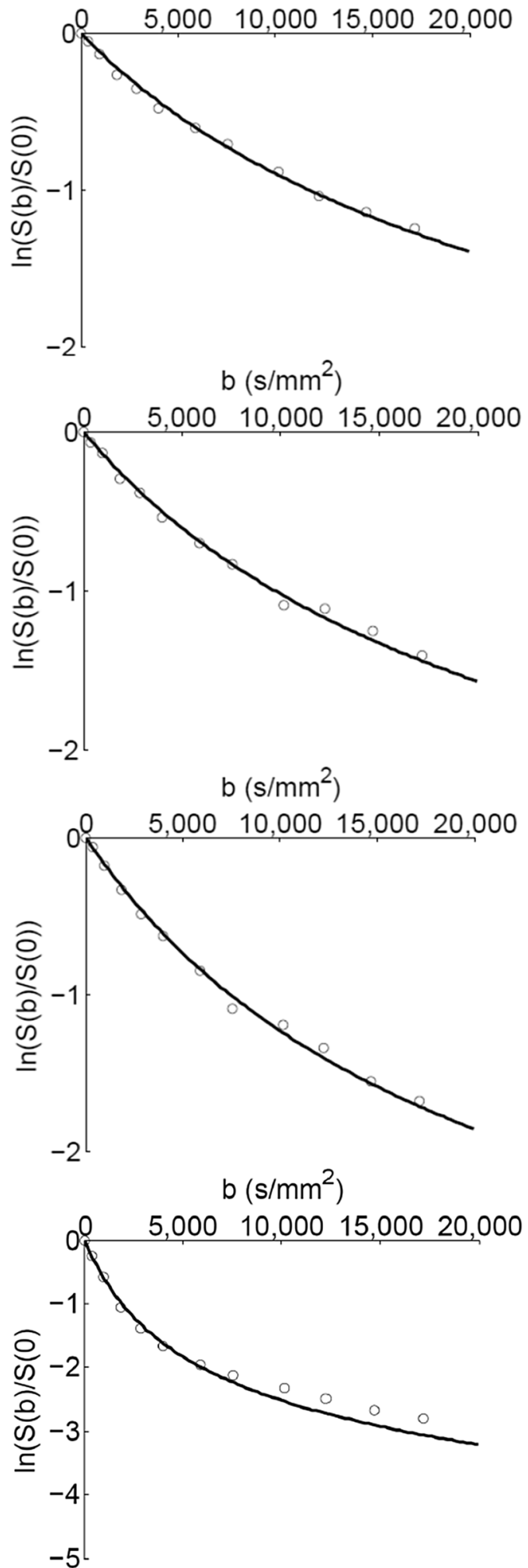

Fig. 3 Example semi-log signal decay plots as a function of diffusion weighting in a white matter (WM) for tCho $(D=0.111$, $\alpha=0.513), \operatorname{tCr}(D=0.124, \alpha=0.562)$, tNAA $(D=0.154, \alpha=0.528)$, and water $(D=0.808, \alpha=0.654)$; and in b gray matter (GM) for tCho
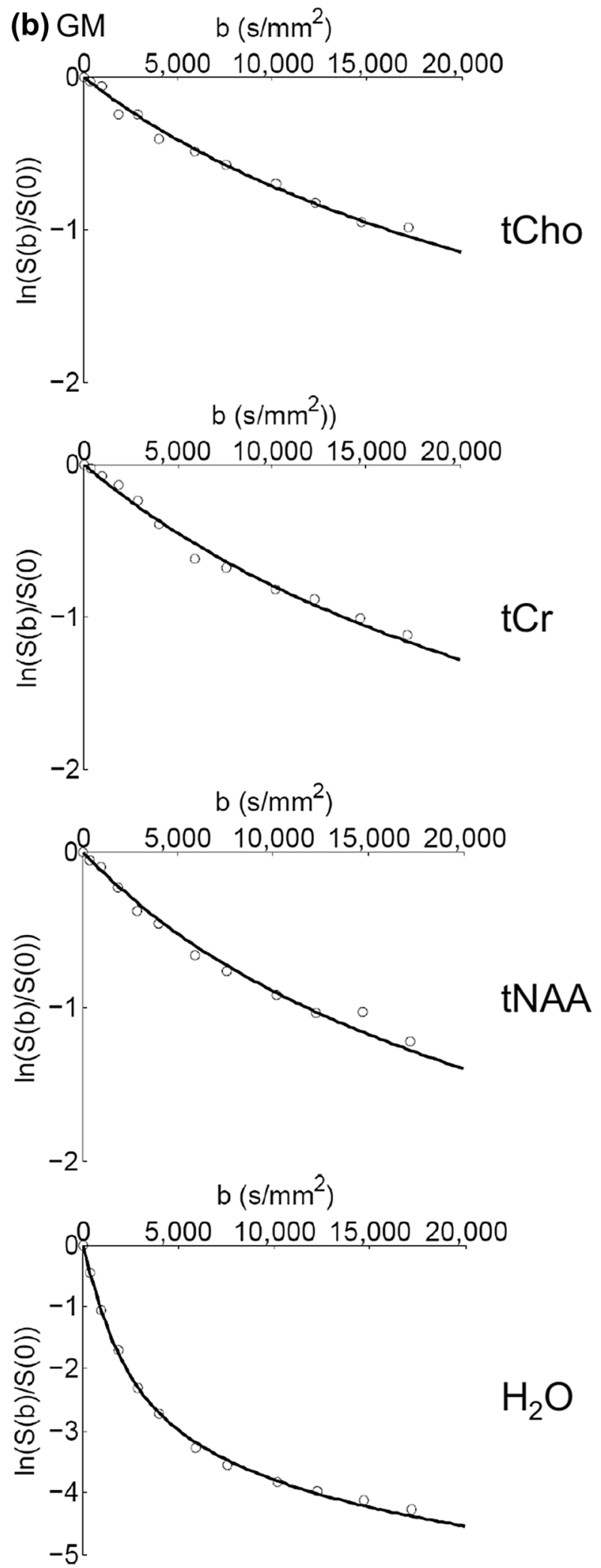

$(D=0.085, \alpha=0.322), \operatorname{tCr}(D=0.093, \alpha=0.461), \mathrm{tNAA}(D=0.115$, $\alpha=0.423)$, and water $(D=1.083, \alpha=0.852)$. Units for $D$ are reported as $\times 10^{-3} \mathrm{~mm}^{2} / \mathrm{s}$. Non-monoexponential behavior is seen in all cases at high $b$ values 
Table 1 Tissue percentages estimated from the segmented $\mathrm{T}_{1}$-weighted anatomical images for the parietal white matter (WM) and occipital gray matter (GM) volumes of interest (VOIs)

\begin{tabular}{lcc}
\hline & Parietal VOI & Occipital VOI \\
\hline WM \% & $72.1 \pm 8.0$ & $28.1 \pm 5.9$ \\
GM \% & $24.4 \pm 5.7$ & $63.6 \pm 5.0$ \\
CSF \% & $3.8 \pm 3.4$ & $8.4 \pm 3.9$ \\
\hline
\end{tabular}
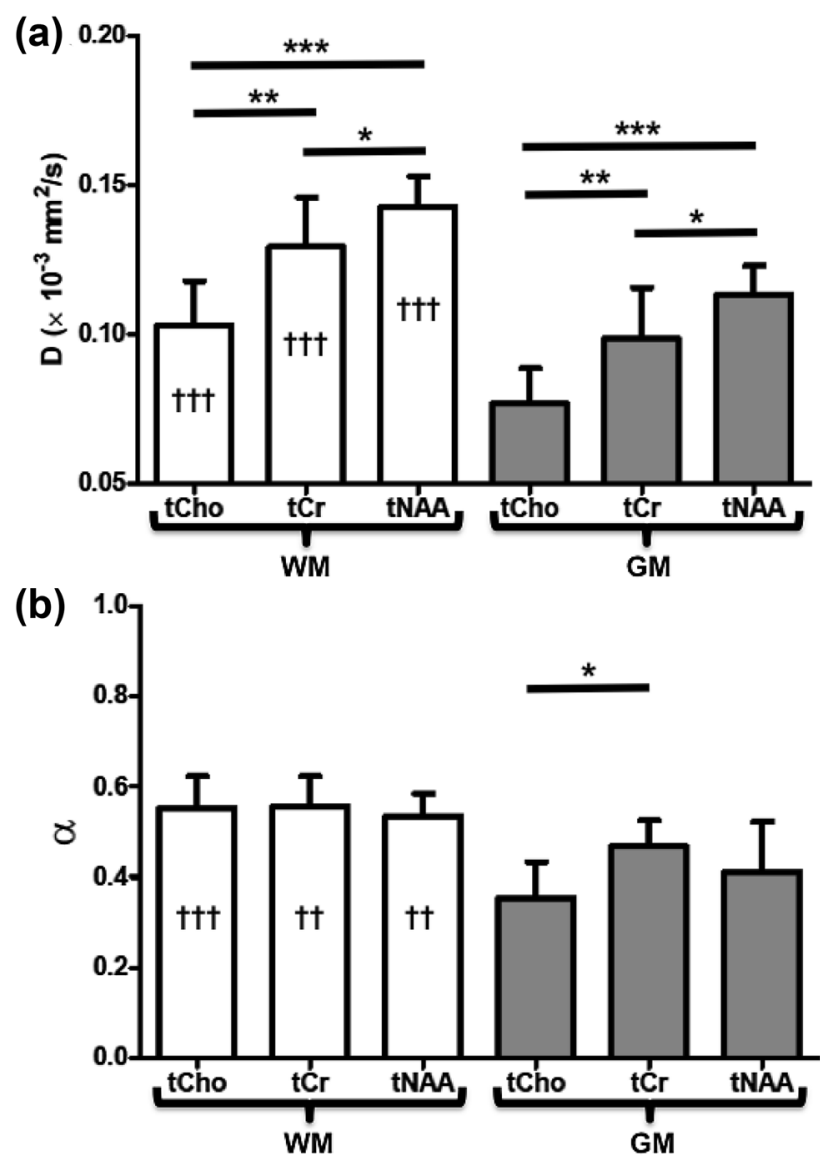

Fig. 4 Metabolite mean and SD for $\mathbf{a} D$ and $\mathbf{b} \alpha$ in the parietal white matter (WM) (left) and occipital gray matter (GM) (right) volumes of interest (VOIs). ${ }^{\dagger}$ Symbol indicates significant difference in individual metabolite values between WM and GM VOIs. *Symbol indicates significant difference in individual metabolite values within each VOI. Symbols are shown for $p<0.05\left({ }^{\dagger}, *\right) p<0.01\left({ }^{\dagger}, * *\right), p<0.001$ $(\dagger \dagger, * * *)$

produced by fitting the data to Eq. 1, clearly indicating deviations from monoexponential behavior for tCho, tCr, tNAA, and water in both the WM and GM VOIs. Figures 4 and 5 report the mean, standard deviation, and statistical analysis (two-way ANOVA with post hoc t-tests, statistical significance threshold $p<0.05$ ) for the estimated values of $D$ and $\alpha$ for the three metabolites and for water in both VOIs. Within the WM ad GM VOIs, the $D$ and $\alpha$ values were tested for the effect of sex, but there were no significant differences between male and female participants (see Supplemental Information for tabular representations of Figs. 4, 5 in S1 Table 1 and S1 Table 2).

\section{Diffusion coefficient, $D$}

Significant differences in $D$ values were found among the metabolites within the same VOI and across tissue types. Within the WM VOI, $D$ (tCho) was significantly lower compared to $D(\mathrm{tCr})$, and $D(\mathrm{tCr})$ was significantly lower than $D$ (tNAA). $D$ (tCho), $D(\mathrm{tCr})$, and $D(\mathrm{tNAA})$ were all significantly lower in GM than in WM. Within the GM VOI, $D$ (tCho) was significantly lower compared to $D(\mathrm{tCr})$, and $D(\mathrm{tCr})$ was significantly lower than $D(\mathrm{tNAA})$. For both the WM and GM VOIs (Figs. 4a, 5), $D\left(\mathrm{H}_{2} \mathrm{O}\right)$ was approximately an order of magnitude higher compared to $D(\mathrm{tCho}), D(\mathrm{tCr})$, and $D$ (tNAA). In contrast to the behavior of the intracellular metabolites, $D\left(\mathrm{H}_{2} \mathrm{O}\right)$ was significantly higher in $\mathrm{GM}$ than in WM.

\section{Subdiffusion index, $a$}

Values of the anomalous subdiffusion index $\alpha$ for the three metabolites were strikingly different between the two brain regions. Within the WM VOI, $\alpha$ (tCho), $\alpha(\mathrm{tCr})$, and $\alpha$ (tNAA) were statistically indistinguishable ( $p=0.671)$ with an average value of $\alpha \sim 0.56$, indicating clear subdiffusion. In GM, $\alpha(\mathrm{tCho}), \alpha(\mathrm{tCr})$, and $\alpha$ (tNAA) were all significantly lower compared to their values in WM. Within the GM VOI, $\alpha$ (tCho) was statistically indistinguishable from $\alpha$ (tNAA) ( $p=0.172)$, and $\alpha(\mathrm{tCr})$ was statistically indistinguishable from $\alpha$ (tNAA) $(p=0.205)$. However, $\alpha$ (tCho) was significantly lower than $\alpha(\mathrm{tCr})(p=0.002)$. As shown in Figs. $4 \mathrm{~b}$ and 5, within both VOIs, $\alpha\left(\mathrm{H}_{2} \mathrm{O}\right)$ was significantly higher compared to $\alpha$ (tCho), $\alpha$ (tCr), and $\alpha$ (tNAA) ( $p=0.008$ for $\mathrm{WM}$ and $p<0.001$ for $\mathrm{GM})$. In contrast to the intracellular metabolite results, $\alpha\left(\mathrm{H}_{2} \mathrm{O}\right)$ was significantly higher in GM than in WM.

\section{High $b$ value water data in WM}

It was observed that the fit of the water data to Eq. 1 in the WM region produced significant residuals at high diffusionweighting. As shown in Fig. 3a (bottom plot) for the water signal attenuation in WM, for $b>6800 \mathrm{~s} / \mathrm{mm}^{2}$ the MittagLeffler function fitting converged on global minima which consistently underestimated the signal amplitude. Across WM, the average maximum difference between the signal amplitude (at $b=17,204 \mathrm{~s} / \mathrm{mm}^{2}$ ) and Eq. 1 was $1.14 \pm 0.47 \%$ of the total signal. This behavior was not observed for the water in GM (Fig. 3b, bottom plot) where the average maximum difference between the signal amplitude and Eq. 1 was 
Fig. 5 Water mean and SD for a $D$ and $\mathbf{b} \alpha$ in the parietal white matter (WM) (left) and occipital gray matter (GM) (right) volumes of interest (VOIs).

${ }^{\dagger}$ Significant differences in water values between $\mathrm{WM}$ and GM VOIs $(p<0.001)$

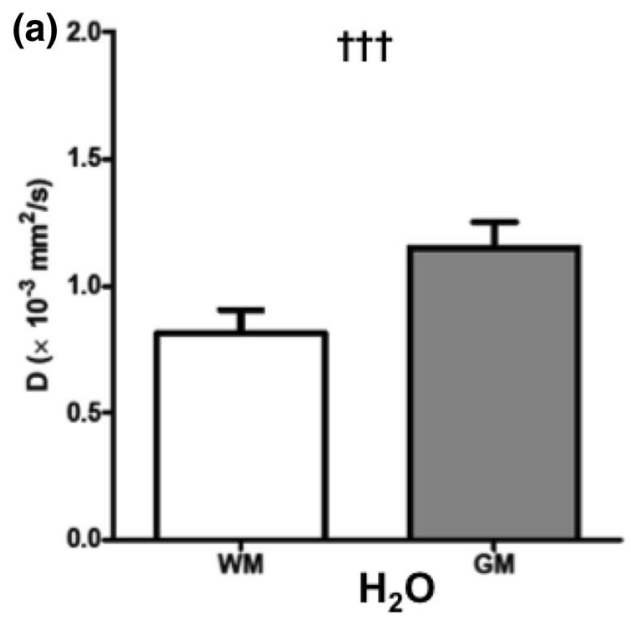

$0.24 \pm 0.38 \%$. Furthermore, the consistent underestimation of the expected signal amplitude was not observed for any of the intracellular metabolites in either WM $(0.13 \pm 0.44 \%)$ or $\mathrm{GM}(0.17 \pm 0.53 \%)$ measurements.

\section{Monte Carlo simulations}

Monte Carlo simulations were performed to verify the experimental results using average group CRLB values reported by LCModel (Provencher 1993) at each diffusionweighted condition. In WM, for example, the average group CRLBs at the highest $b$ value were $7.3 \%$ for tCho, $5.6 \%$ for $\mathrm{tCr}$, and $4.1 \%$ for tNAA. For GM, the average group CRLBs at the highest $b$ value were $9.0 \%$ for tCho, $5.9 \%$ for $\mathrm{tCr}$, and $4.6 \%$ for tNAA. The simulations confirmed the experimental results with one exception: the simulations indicated that there should be a statistically significant difference between $\alpha$ (tCho) and $\alpha$ (tNAA) within GM, but the experimental data did not show this. Based on the CRLB characteristics of tCho and tNAA in GM, $\alpha$ (tCho) converged to $0.38 \pm 0.04$ and $\alpha(\mathrm{tNAA})$ converged to $0.44 \pm 0.04(p=0.04)$ after $N=76$ simulations. In this context, these simulation results would predict that choline exhibits a uniquely low value for $\alpha$ in GM, while all three metabolites share a common higher value for $\alpha$ in WM.

\section{Discussion}

In this work, the cytomorphological complexity of neurons and glia in the human brain was investigated using compartment-specific data acquired with DWS and analyzed in terms of anomalous subdiffusion. The results show that choline, creatine, and $N$-acetyl-aspartate clearly exhibit anomalous subdiffusion $(\alpha<<1)$ in both WM and GM indicating that the diffusion coefficient, $D$, alone is not sufficient in fully capturing the non-monoexponential metabolite signals. In
GM, the intracellular space appeared more heterogeneous and subdiffusive than in WM, opposite to what is observed in water diffusion dynamics. In WM, tCho, $\mathrm{tCr}$, and tNAA are equally subdiffusive although they reside in cells and cellular compartments characterized by widely different morphology. Choline, a predominantly glial metabolite, exhibited the slowest and most heterogeneous subdiffusion in GM compared to WM, indicating a degree of microstructural complexity that is attributed mostly to protoplasmic astrocytes in GM. In the context of the metabolite diffusion dynamics measured here, the water measurements can now also be interpreted in a more meaningful way, and these results support the notion that GM extracellular space is relatively more homogeneous, and exhibits Gaussian diffusion, compared to WM.

\section{Neurons and glia in WM}

For WM, the $D$ values of all three metabolites were widely different, consistent with previous measurements of metabolite diffusivities in WM (Kan et al. 2012). Strikingly, the values of $\alpha$ of all three metabolites in WM were statistically indistinguishable from one another and clearly subdiffusive. The overall microstructural compartmentation experienced by tNAA and tCho is different, yet they exhibit power law dynamics in the same manner at the high range of $b$ values up to $17,000 \mathrm{~s} / \mathrm{mm}^{2}$ where the measurement is sensitized to diffusion at a spatial scale of about $7 \mu \mathrm{m}$. This indicates that although the WM cellular structures in which these metabolites reside are distinctly different, with tNAA almost exclusively in long-range axons and tCho mostly in fibrous astrocytes and oligodendrocytes, these structures share a common microstructural denominator when probed on a spatial scale of a few micrometers (Oberheim et al. 2008; Wilhelmsson et al. 2006). Considering that WM fibrous astrocytes and oligodendrocytes are characterized by long and thin projections with an average total diameters of about $185-200 \mu \mathrm{m}$ 
(Oberheim et al. 2009; Walhovd et al. 2014), these results indicate that intracellular metabolite diffusion, whether in neurons or glia, is predominantly dictated by fiber morphology, and that in WM there is a strong communality between the fibronal structure of axons and projections of fibrous astrocytes and oligodendrocytes (Walhovd et al. 2014). Although these measurements are geared to yield only isotropic measures of metabolite diffusion, it is interesting to note that the overall microstructural and cytosolic properties of both the axonal and glial structures in WM, particularly at high $b$ values, appear similar.

\section{Neurons and glia in GM}

In $\mathrm{GM}$, the values of $D(\mathrm{tNAA}), D(\mathrm{tCr})$, and $D(\mathrm{tCho})$ retain the same order of relative values as in WM, indicating that the dominant factors in determining metabolite displacement are the properties of the cytosolic environment and microstructural complexity. If the source of differences in the values of $D$ among metabolites were to solely reflect cellular size differences, this ordering would not necessarily have been consistent with cellular physiology. For example, based on gross structural considerations alone, one would expect a higher $D$ (tNAA) in GM, as the neuron soma is a larger structure compared to axonal and dendritic projections (Oberheim et al. 2008). Instead, the properties of the cytosol in the neuron soma are evidently different than those in long range axons in a way that indicates the possibility of increased tortuosity, molecular trapping, and organelle density and subcompartmentation in cell bodies (Bates et al. 1996; Patel and Clark 1979). For the predominantly glial metabolite, tCho, differences observed in diffusion dynamics between WM and GM are likely driven by microstructural morphology between fibrous astrocytes and oligodendrocytes, predominantly present in WM, and protoplasmic astrocytes, the most abundant type of astrocyte in GM (Oberheim et al. 2006, 2009, 2012; Wilhelmsson et al. 2006). Interestingly, $\alpha$ (tCho) shows the most significant difference in GM and $\mathrm{WM}$, compared to moderate but significant differences in
$\alpha(\mathrm{tCr})$ and $\alpha(\mathrm{tNAA})$ across the tissue types. Protoplasmic astrocytes are characterized by highly branched processes that occupy a large volume with an average total diameter of about $150 \mu \mathrm{m}$, whereas fibrous astrocytes and oligodendrocytes are characterized by fewer primary processes that are less branched and straighter with an average total diameter of about $185 \mu \mathrm{m}$ (Matyash and Kettenmann 2010; Oberheim et al. 2006, 2009; Wilhelmsson et al. 2006). It should also be noted that in previous studies, WM versus GM differences in $D(\mathrm{tCho}$ ) could not be distinguished based on simple Gaussian diffusion properties (Ercan et al. 2014; Najac et al. 2016), making the anomalous subdiffusion $(\alpha)$ results in this study uniquely important. While tCho is not exclusively confined to glia, but rather is present in astrocytes about two to three times as much compared to neurons (Urenjak et al. 1993), it should be cautioned that there is a neuronal contribution to the tCho results reported here. However, in the context that tCho is the most preferential metabolite for glial cells, this non-invasive imaging method shows the potential to distinguish between the aggregate of fibrous astrocytes and oligodendrocytes in WM both characterized by long, thin and mostly unbranched processes, and the more structurally complex protoplasmic astrocytes in GM based on their respective subdiffusive properties.

\section{High $b$ value measurements and root mean squared displacement}

Figure 6 is an illustrative representation for the link between the fibrous and protoplasmic astrocyte morphological features and the micron-scale spatial sensitization of the diffusion measurement at increasing $b$ values. Over the full range of $b$ values the diffusion measurements probe the cellular microstructure over a scale of approximately $45-7 \mu \mathrm{m}$, with the latter value much lower than typical DWS measurements performed in the past $\left(b<3000 \mathrm{~s} / \mathrm{mm}^{2}, \sim 16 \mu \mathrm{m}\right)$. Furthermore, it is possible to estimate the molecular ensemble of root mean squared displacement (RMSD) for the simple Gaussian case as $\sqrt{2 D t}$, and for the anomalous subdiffusive
Fig. 6 Sketch of a gray matter protoplasmic (left) and a white matter fibrous (right) astrocyte along with the spatial sensitivity $(\mu \mathrm{m})$ of the magnetic resonance diffusion weighting ( $b$ value) protoplasmic astrocyte
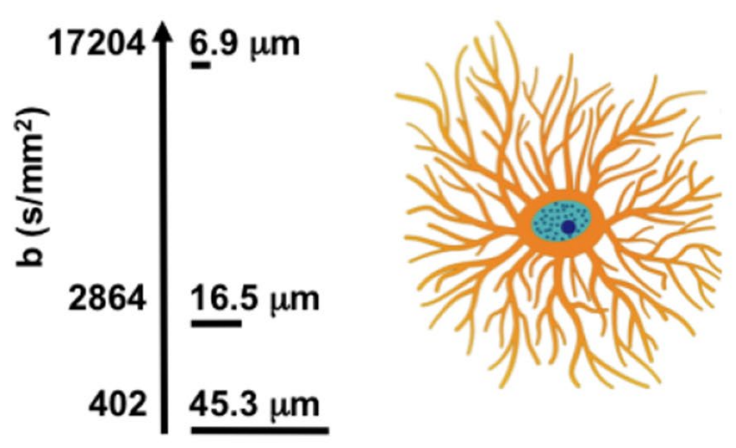

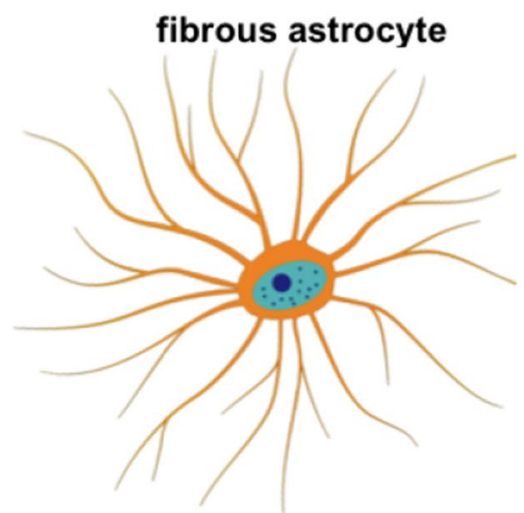


case as $\sqrt{2 D t^{\alpha} / \Gamma(1+\alpha)}$, where $\Gamma$ is the generalized form of the factorial function defined for real numbers (Metzler and Klafter 2000). Taking the mean values of $D$ for water and the three metabolites in GM and WM, the simple Gaussian RMSD for water is about 11.6-13.7 $\mu \mathrm{m}$ and for the metabolites about 3.6-4.9 $\mu \mathrm{m}$. However, by taking the mean values of $D$ and $\alpha$ in GM and WM, respectively, the anomalous subdiffusive RMSD for water is about 5.3-9.6 $\mu \mathrm{m}$ and for the metabolites about $0.9-1.9 \mu \mathrm{m}$. With the additional sensitivity of the anomalous subdiffusion measurements, the fine features between protoplasmic astrocytes in GM with their increased branching, additional endfeet, and shorter projections can be more readily distinguished from the aggregate of fibrous astrocytes and oligodendrocytes in WM, characterized by less branching, thinner projections (Oberheim et al. 2009; Walhovd et al. 2014). Encouragingly, the metabolite anomalous subdiffusive RMSDs (0.9-1.9 $\mu \mathrm{m})$ show excellent agreement in terms of physical displacement with immunofluorescence imaging of individual astrocytic fibers in which the diameter of the thickest process was determined to be no larger than $3 \mu \mathrm{m}$ (Oberheim et al. 2009). This is in contrast to overestimation of the typical fiber process dimensions if simple Gaussian RMSDs (3.6-4.9 $\mu \mathrm{m})$ were to have been used to estimate the metabolite diffusion within these cellular sub-structures. Nevertheless, in either the Gaussian or anomalous framework, these results support the fact that metabolite diffusion offers the opportunity to capture smaller displacements within the fine microstructural and cytosolic properties of neural tissue, which is not possible with the non-specific nature of water diffusion.

\section{Implications for extracellular space in WM and GM}

Considering that the intracellular metabolites reported here do not exchange with the extracellular space that comprised interstitial fluid and the extracellular matrix, the metabolite results together with the water measurements allow for unique analysis of the aggregate intracellular and extracellular properties of WM versus GM. In WM, both $D\left(\mathrm{H}_{2} \mathrm{O}\right)$ and $\alpha\left(\mathrm{H}_{2} \mathrm{O}\right)$ were significantly lower compared to GM, which is opposite to the relative values for the metabolites. Water experiences highly subdiffusive $(\alpha \sim 0.62)$ behavior in WM, not much different from the subdiffusive behavior of the intracellular metabolites in WM ( $\alpha \sim 0.56$ for all three metabolites). Since water is present in the intracellular and extracellular space in comparable fractions, these results suggest that water diffusion in both the intracellular and the extracellular spaces is subdiffusive, and that cross-membrane water exchange rate is slow on the time scale of the diffusion measurement. Slow cross-membrane water exchange in brain WM is possibly due to the high degree of myelination of long range axons in WM tracts (Stanisz et al. 1999), and is supported by in vitro studies in bovine optic nerve (Bjarnason et al. 2005), in rodent spinal cord (Assaf et al. 2000), as well as estimates of apparent cross-membrane exchange rates in the human brain (Nilsson et al. 2013). In GM, the aggregate intracellular and extracellular space experienced by water is significantly less subdiffusive (more Gaussian, $\alpha \sim 0.83$ ) compared to the purely intracellular metabolites $(\alpha \sim 0.35$ to 0.47$)$ and to water diffusion in WM $(\alpha \sim 0.62)$. This result can be interpreted in terms of several different hypotheses: one possibility is that the cross-membrane exchange rate is fast on the time scale of the diffusion time $(\sim 100 \mathrm{~ms})$, perhaps due to higher membrane permeability and a lesser degree of myelination (Nilsson et al. 2013); a second possibility is that the extracellular space is loosely bound with protoplasmic astrocytes arranged in a uniquely non-overlapping manner (Oberheim et al. 2009), thus giving rise to more Gaussian diffusion dynamics in GM. In each of these interpretations, the contribution of the GM extracellular space is significantly decreased at high $b$ values, leaving the highly subdiffusive intracellular space to dominate the diffusion-weighted signal.

\section{High $b$ value water data in WM}

There was consistent underfitting of the data at high $b$ values for the water signal attenuation in WM, a behavior that was not observed in the water signal for GM or in the behavior of the metabolites in either tissue types. This unaccounted extra signal appears to represent a population of water molecules that are bound, or exhibit highly restricted diffusion. Since this pattern was found only in the water of WM, it is possible that this is the residual contribution of water trapped within myelin, or "myelin water" (Mackay et al. 1994). In previous work, the diffusion coefficient of myelin water perpendicular to the myelin sheath in excised frog sciatic nerve measured at a relatively low $b$ value $\left(773 \mathrm{~s} / \mathrm{mm}^{2}\right)$ has been estimated to be about $0.13-0.17 \times 10^{-3} \mathrm{~mm}^{2} / \mathrm{s}$, about 5 times smaller than that of the bulk water in neural WM tissue (Andrews et al. 2006). A more recent study reported a much lower diffusion coefficient for myelin water of $0.0005-0.0012 \times 10^{-3}$ $\mathrm{mm}^{2} / \mathrm{s}$, obtained from numerical simulations based on histological images of excised rat cervical spinal cord (Harkins et al. 2012). The $\mathrm{T}_{2}$ of myelin water (e.g., 10-55 ms) is significantly shorter than that of intra- and extracellular water (Mackay et al. 1994), and so its contribution is expected to be small, perhaps explaining the additional 1\% of WM water signal at the highest diffusion-weighting. The aggregation of these compelling data supports the notion of a small, but highly restricted myelin water component of the MR signal, and further measurements are needed to assess its contribution to diffusion measurements. 


\section{Varying diffusion gradient strength versus varying diffusion time}

In this study, we found a much more significant nonmonoexponential behavior for metabolites than previously reported (Branzoli et al. 2014). This apparent discrepancy emphasizes the need to sample the diffusion decay of metabolite data at high $b$ values $(b>>1 / D)$ to fully capture the phenomenon of subdiffusion. For water subdiffusion, a maximum $b$ value of a few thousands s $/ \mathrm{mm}^{2}$ is enough for a good characterization of the diffusion decay, whereas for metabolites a much higher maximum $b$ value is required.

As this study sampled only one diffusion time with a large range of $b$, it is informative to consider these findings in the context of previous DWS studies in animals which used multiple diffusion times at a fixed low $b$ value (Najac et al. 2014; Palombo et al. 2016). Though the measurement schemes are different, a common aspect in this study and previous works is that the diffusion time ( $>50 \mathrm{~ms}$ ) was sufficiently long such that the measured metabolite displacement had time to interact with the cellular structures that define the boundaries of intracellular space. The ultra-short diffusion time study (Marchadour et al. 2012) fundamentally differs in concept as metabolite displacement is investigated in the regime where free diffusion first begins to interact with obstacles in the cellular structure after a few milliseconds. A key finding in Palombo et al. (2016) is that the time varying properties of metabolite diffusion in experimental data can be used to generate synthetic cellular structures which resemble the complexity of fibrous processes for different types of neuronal and glial cells. Here, the results presented also indicate that the cytosolic properties in the fine processes of neural cells dominate the metabolite displacement as evidenced by the agreement of estimated RMSDs and optical measurements of these typical dimensions. Whether the measurement scheme is chosen to vary either on the spatial or temporal scale, it is clear that metabolite diffusion properties are a function of each variable and it is encouraging that consistent conclusions can be made about microstructural features.

\section{Previous phantom DWS studies, technical limitations, and a note on kurtosis}

To further support the interpretation of these in vivo results, it is important to note that metabolite molecular weight alone does not explain the diffusion trends found in WM and GM, as shown in S1 Table 3 (see Supplemental Information), which summarizes previous measurements in a spherical phantom containing water and metabolites (Ellegood et al. 2005, 2006; Kan et al. 2012). Specifically, the lowest molecular weight metabolite, tCho, has the highest in vitro diffusion coefficient compared to both the creatine and NAA compounds, which is opposite to the in vivo results in WM and GM in this study. Rather, the results in this current study show that metabolite diffusion dynamics are largely determined by cytoplasmic properties (i.e., tortuosity, crowding), transient molecular trapping and binding to the cytoskeleton, and heterogeneity in intracellular microstructural architecture, all of which reduce the order of the bulk diffusion coefficients by about $5-10$-fold from in vitro to in vivo (Valette et al. 2007).

A technical limitation of this study is that the relatively large size of the VOIs limited the ability to choose VOIs with either purely WM or GM. This was primarily due to SNR considerations, in particular at very high $b$ values, which are important for the characterization of metabolite subdiffusion. It is expected that future studies which combine stronger gradients (allowing shorter echo times and a lower signal loss due to $\mathrm{T}_{2}$ relaxation) together with the use of parallel transmit will allow smaller VOIs to be investigated, reducing the effects of partial volumes in WM and GM. Previous DWS studies in animals (Marchadour et al. 2012; Najac et al. 2014; Palombo et al. 2016) have not tried to distinguish between differences WM and GM, mostly due to limitations in spatial resolution given the size of the brain structures in smaller animals. Therefore, DWS studies in humans at $7 \mathrm{~T}$ offer a unique opportunity to study cytomorphology in the human brain with high specificity and future studies performed with state-of-the-art hardware and pulse sequences have the potential to further elucidate the findings presented in the current work. Subsequently, these techniques in this study could be further adapted for clinical relevance in $3 \mathrm{~T}$ systems as there is precedence that reproducible and optimized DWS studies can be practically performed on both 7 and $3 \mathrm{~T}$ systems (Wood et al. 2015).

Finally, it should be noted that estimates of the subdiffusion index, $\alpha$, can be converted to a kurtosis value through the mathematical relationship derived in (Ingo et al. 2015), however, the traditional method of estimating kurtosis using a fit to the Taylor series expansion (Jensen et al. 2005) is not applicable for these high $b$ data as the Taylor series model is only stable and monotonic for a limited range of diffusion weightings (see (Ingo et al. 2015) for treatment).

\section{Conclusions}

Previous studies using optical imaging have shown that individually traced particles follow power law anomalous subdiffusive dynamics in the presence of intracellular biological environments (Banks and Fradin 2005; Ghosh et al. 2015; Weiss et al. 2004). In this study, using non-invasive DWS in the healthy in vivo human brain, it has been shown that within the intracellular environment encapsulated by neuronal and glial compartments, entire distributions of molecules also undergo displacement in a manner that is 
consistent with power law anomalous subdiffusion. The unprecedented in vivo measurements in this study enable further insight into WM and GM microstructure, both from an intracellular and extracellular perspective, by providing new evidence of anomalous subdiffusive dynamics in bulk tissue microstructure. This emerging technique not only has the potential to further understand the healthy human brain, but also to better characterize neuropathophysiological processes, ranging from inflammation to glioma classification (Ercan et al. 2016; Sui et al. 2016).

Acknowledgements The authors acknowledge special gratitude to Marjolein Bulk for recruitment of the study participants, Gerrit Kracht for the illustrations of glial cells, and to all study participants for their patience and perseverance.

Funding This work has been supported by a grant from the Whitaker International Program of the Institute of International Education. Additional funding was provided by the European Research Council (ERC ADC 670629 NOMA MRI).

\section{Compliance with ethical standards}

Conflict of interest The authors declare that they have no conflict of interest.

Ethical approval The study adhered to the Leiden University Medical Center Institutional Review Board guidelines.

Informed consent Informed consent was obtained from all individual participants included in the study.

Open Access This article is distributed under the terms of the Creative Commons Attribution 4.0 International License (http://creativeco mmons.org/licenses/by/4.0/), which permits unrestricted use, distribution, and reproduction in any medium, provided you give appropriate credit to the original author(s) and the source, provide a link to the Creative Commons license, and indicate if changes were made.

\section{References}

Andrews TJ, Osborne MT, Does MD (2006) Diffusion of myelin water. Magn Reson Med 56:381-385. https://doi.org/10.1002/ mrm. 20945

Assaf Y, Basser PJ (2005) Composite hindered and restricted model of diffusion (CHARMED) MR imaging of the human brain. Neuroimage 27:48-58

Assaf Y, Cohen Y (1998a) In vivo and in vitro bi-exponential diffusion of $N$-acetyl aspartate (NAA) in rat brain: a potential structural probe? NMR Biomed 11:67-74. https://doi. org/10.1002/(SICI)1099-1492(199804)11:2\%3C67::AID-NBM50 3\%3E3.0.CO;2-5

Assaf Y, Cohen Y (1998b) Non-mono-exponential attenuation of water and $\mathrm{n}$-acetyl aspartate signals due to diffusion in brain tissue. $\mathrm{J}$ Magn Reson 131:69-85

Assaf Y, Mayk A, Cohen Y (2000) Displacement imaging of spinal cord using q-space diffusion-weighted MRI. Magn Reson
Med 44:713-722. https://doi.org/10.1002/1522-2594(20001 1) 44:5\%3C713::Aid-Mrm9\%3E3.0.Co;2-6

Banks DS, Fradin C (2005) Anomalous diffusion of proteins due to molecular crowding. Biophys J 89:2960-2971

Barkai E, Garini Y, Metzler R (2012) Strange kinetics of single molecules in living cells. Phys Today 65:29-35

Bates TE, Strangward M, Keelan J, Davey GP, Munro PM, Clark JB (1996) Inhibition of $\mathrm{N}$-acetylaspartate production: implications for 1H MRS studies in vivo. Neuroreport 7:1397-1400

Bjarnason TA, Vavasour IM, Chia CLL, MacKay AL (2005) Characterization of the NMR behavior of white matter in bovine brain. Magn Reson Med 54:1072-1081. https://doi.org/10.1002/ mrm. 20680

Brandejsky V, Boesch C, Kreis R (2014) Proton diffusion tensor spectroscopy of metabolites in human muscle in vivo. Magn Reson Med. https://doi.org/10.1002/mrm.25139

Branzoli F, Ercan E, Webb A, Ronen I (2014) The interaction between apparent diffusion coefficients and transverse relaxation rates of human brain metabolites and water studied by diffusion-weighted spectroscopy at 7 T. NMR Biomed 27:495-506. https://doi. org/10.1002/nbm.3085

Brink WM, van der Jagt AMA, Versluis MJ, Verbist BM, Webb AG (2014) High permittivity dielectric pads improve high spatial resolution magnetic resonance imaging of the inner ear at $7 \mathrm{~T}$. Investig Radiol 49:271-277

Cao P, Wu EX (2016) In vivo diffusion MRS investigation of non-water molecules in biological tissues. NMR Biomed 30:e3481

Choi JK, Dedeoglu A, Jenkins BG (2007) Application of MRS to mouse models of neurodegenerative illness. NMR Biomed. https ://doi.org/10.1002/nbm.1145

Cotts RM, Hoch MJR, Sun T, Markert JT (1989) Pulsed field gradient stimulated echo methods for improved NMR diffusion measurements in heterogeneous systems. J Magn Reson 83:252-266

de Graaf RA, van Kranenburg A, Nicolay K (2000) In vivo (31)P-NMR diffusion spectroscopy of ATP and phosphocreatine in rat skeletal muscle. Biophys J 78:1657-1664. https://doi.org/10.1016/S0006 $-3495(00) 76717-8$

Deelchand DK, Auerbach EJ, Marjanska M (2017) Apparent diffusion coefficients of the five major metabolites measured in the human brain in vivo at 3 T. Magn Reson Med. https://doi.org/10.1002/ mrm.26969

Dreher W, Busch E, Leibfritz D (2001) Changes in apparent diffusion coefficients of metabolites in rat brain after middle cerebral artery occlusion measured by proton magnetic resonance spectroscopy. Magn Reson Med 45:383-389

Ellegood J, Hanstock CC, Beaulieu C (2005) Trace apparent diffusion coefficients of metabolites in human brain using diffusion weighted magnetic resonance spectroscopy. Magn Reson Med 53:1025-1032

Ellegood J, Hanstock CC, Beaulieu C (2006) Diffusion tensor spectroscopy (DTS) of human brain. Magn Reson Med 55:1-8. https ://doi.org/10.1002/mrm.20777

Ercan AE, Techawiboonwong A, Versluis MJ, Webb AG, Ronen I (2014) Diffusion-weighted chemical shift imaging of human brain metabolites at 7 T. Magn Reson Med 82:2011-2011. https://doi. org $/ 10.1002 / \mathrm{mrm} .25346$

Ercan E et al (2016) Glial and axonal changes in systemic lupus erythematosus measured with diffusion of intracellular metabolites. Brain. https://doi.org/10.1093/brain/aww031

Ghosh SK, Cherstvy AG, Grebenkov DS, Metzler R (2015) Anomalous, non-Gaussian tracer diffusion in heterogeneously crowded environments. arXiv: 150802029

Hall MG, Barrick TR (2008) From diffusion-weighted MRI to anomalous diffusion imaging. Magn Reson Med 59:447-455

Harkins KD, Dula AN, Does MD (2012) Effect of intercompartmental water exchange on the apparent myelin water fraction 
in multiexponential T2 measurements of rat spinal cord. Magn Reson Med 67:793-800

Hayakawa K et al (2016) Transfer of mitochondria from astrocytes to neurons after stroke. Nature 535:551-555. https://doi.org/10.1038/ nature 18928

Ingo C, Magin RL, Colon-Perez L, Triplett W, Mareci TH (2014a) On random walks and entropy in diffusion-weighted magnetic resonance imaging studies of neural tissue. Magn Reson Med 71:617-627

Ingo C, Magin RL, Parrish TB (2014b) New insights into the fractional order diffusion equation using entropy and kurtosis. Entropy 16:5838-5852

Ingo C, Sui Y, Chen Y, Parrish T, Webb A, Ronen I (2015) Parsimonious continuous time random walk models and kurtosis for diffusion in magnetic resonance of biological tissue. Front Phys. https ://doi.org/10.3389/fphy.2015.00011

Ingo C, Barrick TR, Webb AG, Ronen I (2017) Accurate padé global approximations for the mittag-leffler function, its inverse, and its partial derivatives to efficiently compute convergent power series international. J Appl Comput Math 3:347-362

Jenkinson M, Beckmann CF, Behrens TE, Woolrich MW, Smith SM (2012) FSL. Neuroimage 62:782-790. https://doi.org/10.1016/j. neuroimage.2011.09.015

Jensen JH, Helpern JA, Ramani A, Lu H, Kaczynski K (2005) Diffusional kurtosis imaging: the quantification of non-gaussian water diffusion by means of magnetic resonance imaging. Magn Reson Med 53:1432-1440

Jones DK (2010) Diffusion MRI: theory, methods, and application. Oxford University Press, Oxford

Kan HE et al (2012) Differences in apparent diffusion coefficients of brain metabolites between grey and white matter in the human brain measured at 7 T. Magn Reson Med 67:1203-1209

Kroenke CD, Ackerman JJ, Yablonskiy DA (2004) On the nature of the NAA diffusion attenuated MR signal in the central nervous system. Magn Reson Med 52:1052-1059. https://doi.org/10.1002/ mrm. 20260

Le Bihan D, Moonen CTW, van Zijl PCM, Pekar J, DesPres D (1991) Measuring random microscopic motion of water in tissues with MR imaging: a cat brain study. J Comput Assist Tomogr 15:19-25

Le Belle JEL, Harris NG, Williams SR, Bhakoo KK (2002) A comparison of cell and tissue extraction techniques using high-resolution 1 H-NMR spectroscopy. NMR Biomed 15:37-44

Mackay A, Whittall K, Adler J, Li D, Paty D, Graeb D (1994) In vivo visualization of myelin water in brain by magnetic resonance. Magn Reson Med 31:673-677

Magin RL, Abdullah O, Baleanu D, Zhou XJ (2008) Anomalous diffusion expressed through fractional order differential operators in the Bloch-Torrey equation. J Magn Reson 190:255-270

Marchadour C, Brouillet E, Hantraye P, Lebon V, Valette J (2012) Anomalous diffusion of brain metabolites evidenced by diffusionweighted magnetic resonance spectroscopy in vivo. J Cereb Blood Flow Metab 32:2153-2160

Matyash V, Kettenmann H (2010) Heterogeneity in astrocyte morphology and physiology. Brain Res Rev 63:2-10. https://doi. org/10.1016/j.brainresrev.2009.12.001

Merboldt KD, Horstermann D, Hanicke W, Bruhn H, Frahm J (1993) Molecular self-diffusion of intracellular metabolites in rat brain in vivo investigated by localized proton NMR diffusion spectroscopy. Magn Reson Med 29:125-129

Metzler R, Klafter J (2000) The random walk's guide to anomalous diffusion: a fractional dynamics approach. Phys Rep 339:1-77

Mittag-Leffler GM (1903) Sur la nouvelle fonction E $\alpha$. (x). CR Acad Sci Paris 137:554-558

Mittag-Leffler G (1905) Sur la representation analytique d'une branche uniforme d'une fonction monogene. Acta Math 29:101-181
Mobius W, Nave KA, Werner HB (2016) Electron microscopy of myelin: structure preservation by high-pressure freezing. Brain Res 1641:92-100. https://doi.org/10.1016/j.brainres.2016.02.027

Moonen CT, van Zijl PC, Le Bihan D, DesPres D (1990) In vivo NMR diffusion spectroscopy: 31P application to phosphorus metabolites in muscle. Magn Reson Med 13:467-477

Najac C et al (2014) Intracellular metabolites in the primate brain are primarily localized in long fibers rather than in cell bodies as shown by diffusion-weighted magnetic resonance spectroscopy. NeuroImage 90:374-380. https://doi.org/10.1016/j.neuroimage .2013.12.045

Najac C, Branzoli F, Ronen I, Valette J (2016) Brain intracellular metabolites are freely diffusing along cell fibers in grey and white matter, as measured by diffusion-weighted MR spectroscopy in the human brain at 7 T. Brain Struct Funct 221:1245-1254. https ://doi.org/10.1007/s00429-014-0968-5

Nehrke K, Börnert P (2012) DREAM-a novel approach for robust, ultrafast, multislice B1 mapping. Magn Reson Med 68:1517-1526

Nicolay K, Braun KPJ, De Graaf RA, Dijkhuizen RM, Kruiskamp MJ (2001) Diffusion NMR spectroscopy. NMR Biomed 14:94-111

Nilsson M, Lätt J, Van Westen D, Brockstedt S, Lasič S, Ståhlberg F, Topgaard D (2013) Noninvasive mapping of water diffusional exchange in the human brain using filter-exchange imaging. Magn Reson Med 69:1573-1581

Oberheim NA, Wang X, Goldman S, Nedergaard M (2006) Astrocytic complexity distinguishes the human brain. Trends Neurosci 29:547-553

Oberheim NA, Tian G-F, Han X, Peng W, Takano T, Ransom B, Nedergaard M (2008) Loss of astrocytic domain organization in the epileptic brain. J Neurosci 28:3264-3276

Oberheim NA et al (2009) Uniquely hominid features of adult human astrocytes. J Neurosci 29:3276-3287

Oberheim NA, Goldman SA, Nedergaard M (2012) Heterogeneity of astrocytic form and function. Methods Mol Biol 814:23-45

Özarslan E, Shepherd TM, Koay CG, Blackband SJ, Basser PJ (2012) Temporal scaling characteristics of diffusion as a new MRI contrast: findings in rat hippocampus. Neuroimage 60:1380-1393

Palombo M et al (2016) New paradigm to assess brain cell morphology by diffusion-weighted MR spectroscopy in vivo. Proc Natl Acad Sci USA 113:6671-6676. https://doi.org/10.1073/pnas.15043 27113

Palombo M, Shemesh N, Ronen I, Valette J (2017) Insights into brain microstructure from in vivo DW-MRS. Neuroimage. https://doi. org/10.1016/j.neuroimage.2017.11.028

Pannese E (2015) Neurocytology: fine structure of neurons, nerve processes, and neuroglial cells. Springer, Berlin

Pasternak O, Sochen N, Gur Y, Intrator N, Assaf Y (2009) Free water elimination and mapping from diffusion MRI. Magn Reson Med 62:717-730. https://doi.org/10.1002/mrm.22055

Patel TB, Clark JB (1979) Synthesis of N-acetyl-L-aspartate by rat brain mitochondria and its involvement in mitochondrial/cytosolic carbon transport. Biochem J 184:539-546

Pfeuffer J, Tkac I, Gruetter R (2000) Extracellular-intracellular distribution of glucose and lactate in the rat brain assessed noninvasively by diffusion-weighted $1 \mathrm{H}$ nuclear magnetic resonance spectroscopy in vivo. J Cereb Blood Flow Metab 20:736-746. https://doi.org/10.1097/00004647-200004000-00011

Pierpaoli C, Jezzard P, Basser PJ, Barnett A, Di Chiro G (1996) Diffusion tensor MR imaging of the human brain. Radiology 201:637-648

Posse S, Cuenod CA, Le Bihan D (1993) Human brain: proton diffusion. MR Spectrosc Radiol 188:719-725

Provencher SW (1993) Estimation of metabolite concentrations from localized in vivo proton NMR spectra. Magn Reson Med 30:672-679 
Ronen I, Ercan E, Webb A (2013) Axonal and glial microstructural information obtained with diffusion-weighted magnetic resonance spectroscopy at 7 T. Front Integr Neurosci 7:13-13. https://doi. org/10.3389/fnint.2013.00013

Ronen I, Budde M, Ercan E, Annese J, Techawiboonwong A, Webb A (2014) Microstructural organization of axons in the human corpus callosum quantified by diffusion-weighted magnetic resonance spectroscopy of $\mathrm{N}$-acetylaspartate and post-mortem histology. Brain Struct Funct 219:1773-1785

Shemesh N, Rosenberg JT, Dumez J-N, Muniz JA, Grant SC, Frydman L (2014) Metabolic properties in stroked rats revealed by relaxation-enhanced magnetic resonance spectroscopy at ultrahigh fields. Nat Commun 5:4958-4958. https://doi.org/10.1038/ ncomms5958

Stanisz GJ, Kecojevic A, Bronskill MJ, Henkelman RM (1999) Characterizing white matter with magnetization transfer and $\mathrm{T}_{2}$. Magn Reson Med 42:1128-1136. https://doi.org/10.1002/(SICI)15222594(199912)42:6\%3C1128::AID-MRM18\%3E3.0.CO;2-9

Stejskal EO, Tanner JE (1965) Spin diffusion measurements spin echoes in the presence of a time-dependent field gradient. J Chem Phys 42:288-292

Sui Y, Xiong Y, Jiang J, Karaman MM, Xie KL, Zhu W, Zhou XJ (2016) Differentiation of low-and high-grade gliomas using high b-value diffusion imaging with a non-gaussian diffusion model. Am J Neuroradiol. https://doi.org/10.3174/ajnr.A4836

Teeuwisse WM, Brink WM, Haines KN, Webb AG (2012) Simulations of high permittivity materials for $7 \mathrm{~T}$ neuroimaging and evaluation of a new barium titanate-based dielectric. Magn Reson Med 67:912-918

Upadhyay J, Hallock K, Erb K, Kim D-S, Ronen I (2007) Diffusion properties of NAA in human corpus callosum as studied with diffusion tensor spectroscopy. Magn Reson Med 58:1045-1053

Upadhyay J, Hallock K, Ducros M, Kim DS, Ronen I (2008) Diffusion tensor spectroscopy and imaging of the arcuate fasciculus. Neuroimage 39:1-9. https://doi.org/10.1016/j.neuroimage.2007.08.046

Urenjak J, Williams SR, Gadian DG, Noble M (1993) Proton nuclear magnetic resonance spectroscopy unambiguously identifies different neural cell types. J Neurosci 13:981-989
Valette J, Guillermier M, Besret L, Hantraye P, Bloch G, Lebon V (2007) Isoflurane strongly affects the diffusion of intracellular metabolites, as shown by $1 \mathrm{H}$ nuclear magnetic resonance spectroscopy of the monkey brain. J Cereb Blood Flow Metab 27:588-596

van Doorn A, Bovendeerd PH, Nicolay K, Drost MR, Janssen JD (1996) Determination of muscle fibre orientation using diffusionweighted MRI. Eur J Morphol 34:5-10

van der Toorn A, Dijkhuizen RM, Tulleken CA, Nicolay K (1996) Diffusion of metabolites in normal and ischemic rat brain measured by localized 1H MRS. Magn Reson Med 36:914-922

Versluis MJ, Kan HE, van Buchem MA, Webb AG (2010) Improved signal to noise in proton spectroscopy of the human calf muscle at 7 T using localized B1 calibration. Magn Reson Med 63:207-211. https://doi.org/10.1002/mrm.22195

Walhovd KB, Johansen-Berg H, Karadottir RT (2014) Unraveling the secrets of white matter-bridging the gap between cellular, animal and human imaging studies. Neuroscience 276:2-13. https://doi. org/10.1016/j.neuroscience.2014.06.058

Webb AG, Collins CM (2010) Parallel transmit and receive technology in high-field magnetic resonance neuroimaging. Int J Imag Syst Tech 20:2-13. https://doi.org/10.1002/ima.20219

Weiss M, Elsner M, Kartberg F, Nilsson T (2004) Anomalous subdiffusion is a measure for cytoplasmic crowding in living cells. Biophys J 87:3518-3524

Wick M, Nagatomo Y, Prielmeier F, Frahm J (1995) Alteration of intracellular metabolite diffusion in rat brain in vivo during ischemia and reperfusion. Stroke 26:1930-1933 (discussion 1934)

Wilhelmsson U et al (2006) Redefining the concept of reactive astrocytes as cells that remain within their unique domains upon reaction to injury. Proc Natl Acad Sci USA 103:17513-17518

Wood ET, Ercan AE, Branzoli F, Webb A, Sati P, Reich DS, Ronen I (2015) Reproducibility and optimization of in vivo human diffusion-weighted MRS of the corpus callosum at $3 \mathrm{~T}$ and $7 \mathrm{~T}$. NMR Biomed 28:976-987. https://doi.org/10.1002/nbm.3340

Xiao F, Hrabe J, Hrabetova S (2015) Anomalous extracellular diffusion in rat cerebellum. Biophys J 108:2384-2395 